\title{
Miller Fisher variant of Guillain Barre Syndrome: A Case Report
}

\author{
R Saha ${ }^{1}$, SK Saha ${ }^{2}$, N Islam³, R Kabir ${ }^{4}, \mathrm{~A} \mathrm{Mondal}^{5}, \mathrm{AA} \mathrm{Mamun}^{6}$, AK Saha
}

\begin{abstract}
:
Miller Fisher Syndrome (MFS) is characterized by acute onset of ophthalmoplegia, ataxia and loss of tendon reflexes with relative sparing of strength in the extremities and trunk. Our patient Mr. Akter Sheikh, 40 years male, non diabetic, non hypertensive, presented with generalized weakness and difficulty in walking for 7 days preceded by a history of respiratory tract infection. After clinical and CSF examination, he was diagnosed as a case of Miller-Fisher syndrome. With treatment he improved significantly.
\end{abstract}

Key words : Guillain-Barre Syndrome, Miller Fisher Syndrome, ophthalmoplegia, ataxia, areflexia.

\section{Introduction :}

Guillain-Barre Syndrome (GBS), also known as acute idiopathic polyneuritis has several variants. These variants all share similar patterns of symptoms and prognosis. Miller Fisher Syndrome (MFS) is a rare variant of GBS, observed in only about $1 \%$ to $5 \%$ of all cases of GBS in western countries. It occurs more in men than women by a ratio of approximately $2: 1$. The mean age of onset of MFS is 43.6 years, though onset has been documented in individuals between the ages of 13 and 78 years $^{1-3}$.

Miller Fisher Syndrome (MFS) of GBS, an unusual axonal variant is characterized by acute onset of ophthalmoplegia, ataxia and loss of deep tendon

1.Dr. Radheshyam Saha, MBBS, FCPS (Medicine), MD (Neurology), Associate Professor \& Head, Department of Neuromedicine, Faridpur Medical College, Faridpur.

2. Dr. Suranjit Kumar Saha, MBBS, Medical Officer, Department of Medicine, Faridpur Medical College \& Hospital, Faridpur.

3. Dr. Muhammad Nurul Islam, MBBS, Registrar, Department of Medicine, Faridpur Medical College \& Hospital, Faridpur.

4. Dr. Mohammad Rafiqul Kabir, MBBS, D-card, Assistant Registrar, Department of Neuromedicine, Faridpur Medical College \& Hospital, Faridpur.

5. Dr. Aniruddha Mondal, MBBS, Registrar, Department of Physical Medicine, Faridpur Medical College \& Hospital, Faridpur.

6. Dr. Abdullah Al Mamun, MBBS, Assistant Registrar, Department of Medicine, Faridpur Medical College \& Hospital, Faridpur.

7. Dr. Aloke Kumar Saha, FCPS (Paed), Associate professor, Dept. of paediatrics, FMC, Faridpur.

Address of correspondence :

Dr. Radheshyam Saha, MBBS, FCPS (Medicine), MD (Neurology),

Associate Professor \& Head, Dept. of Neurology, Faridpur

Medical College, Faridpur. Phone: +88-01711883080,

Email:drrsaha@yahoo.com reflexes. Patients present with diplopia followed by gait and limb ataxia. Ocular signs include complete ophthalmoplegia, dilated non reactive pupils and external ophthalmoplegia with or without ptosis. The ataxia is produced by peripheral sensory nerve dysfunction and not by cerebellar injury. Facial weakness and sensory loss may also occur. The process is mediated by autoantibodies directed against component of myelin found in peripheral nerves ${ }^{4}$.

The main difference between MFS and more common variants of GBS is that the first nerve groups to be affected by paralysis in patients with MFS are those in the head, resulting in difficulty in controlling eye muscles and balance. Paralysis in other forms of GBS typically begins in the legs ${ }^{1-3}$. On examination, the patients show signs of ophthalmoplegia, bilateral hypo or areflexia, normal muscle strength, absent plantar response and intact sensation. The investigations are analysis of cerebrospinal fluid (CSF) consistent with albuminocytological dissociation and raised GQIb ganglioside immunoglobulin $\mathrm{G}$ ( $\mathrm{IgG})$. Electrodiagnostic studies demonstrate an axonal process affecting predominantly sensory nerve fibers with only mild motor nerve conduction abnormalities. Sensory nerve action potential (SNAP) amplitude is reduced or absent. Motor conduction studies, including F-wave latencies, are usually normal ${ }^{5}$. Anti-GQIb antibodies activated by certain strains of Campylobacter jejuni have a relatively high specificity and sensitivity for $\mathrm{MFS}^{2}$. Dense concentrations of GQIb ganglioside are found in the oculomotor nerve (cranial nerve III), trochlear nerve (cranial nerve IV) and abducens nerve (cranial nerve VI) of patients with MFS, which may explain the relationship between anti-GQIb antibodies and ophthalmoplegia ${ }^{3,6,7}$. Titers of anti-GQIb antibodies in CSF greater than 1: 40 are specific for $\mathrm{MFS}^{3}$. 
Onset of recovery from ataxia and ophthalmoplegia in patients ranges from approximately 2 weeks to 2 months ${ }^{6}$, with a mean onset time at 10 weeks ${ }^{3}$. Most patients feel well or recover from ataxia and ophthalmoplegia as well as areflexia within 6 weeks.

\section{Case report:}

Mr. Akter Sheikh, a 40 year male patient, admitted with generalized weakness and difficulty in walking for 7 days which develop gradually, preceded by a history of upper respiratory tract infection about 12 days back. He had no respiratory difficulty or sphincteric disturbance. On examination, his vital signs were normal but neurologically he had complete ophthalmoplegia bilaterally and right sided facial nerve palsy. His motor function revealed that bulks of the muscles were normal on both sides, tone was reduced, muscle power was reduced $(3 / 5$ on both upper limbs, $4 / 5$ on both lower limbs). Reflexes markedly reduced, planter showed no response on both sides. It was not possible to examine gait, as patient was bed ridden. All modalities of sensory function were intact. Signs of meningeal irritation were absent.

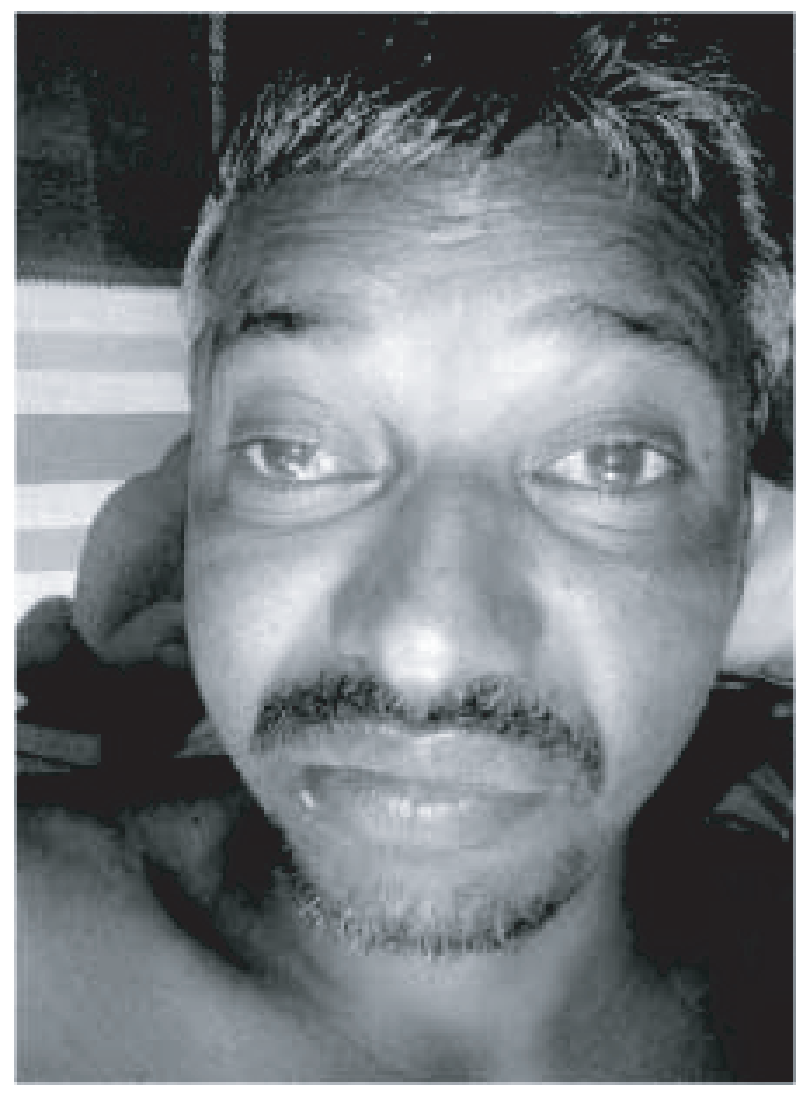

Figure-1: Patient with bilateral ophthalmoplegia and right sided facial palsy.
His investigations revealed; CSF study: Colour - crystal clear, glucose - $83 \mathrm{mg} / \mathrm{dl}$, Protein - $70 \mathrm{mg} / \mathrm{dl}$, cell count -WBC-03/cmm (predominantly lymphocytes), bacteriology - normal (Gram stain- no organism, no AFB). Nerve Conduction Study - not done (as not available in Faridpur). $C B C, R B S$, s. creatinine and $s$. electrolytes were normal. A diagnosis of Miller-Fisher syndrome was made clinically and by laboratory findings (CSF study).

\section{Discussion:}

MFS (Miller-Fisher Syndrome) is a rare case representing about $1-5 \%$ of all cases of GBS. MFS is diagnosed by clinical features, CSF findings, nerve conduction velocity (NCV) and raised serum GQIb ganglioside antibody level. Here, typical clinical findings and albuminocytological dissociation in the CSF help in the diagnosis of MFS. In this case electrophysiological studies and serum GQIb were not done due to lack of facility. Anti-GQIb IgG antibodies are found in $>90 \%$ of patients with MFS and titers of IgG are highest early in the course. Anti-GQIb IgG antibodies are not found in other forms of GBS unless there is extraocular motor nerve involvement. Electrodiagnostic features may be minimal and the CSF protein level may not rise until the end of the first week. If the diagnosis is strongly suspected, treatment should be initiated without waiting for characteristic electrodiagnostic and CSF findings.

\section{Conclusion:}

Miller-Fisher Syndrome is an uncommon variant of Guillain - Barre Syndrome that can be diagnosed clinically by its characteristic triad of featuresareflexia, ataxia and opthalmoplegia. The prognosis for most individuals is good. Early diagnosis and management reduce the morbidity and mortality.

\section{References :}

1. Fisher M. An Unusual Variant of Acute Idiopathic Polyneuritis (Syndrome of Opthalmoplegia, Ataxia and Areflexia). N Engl J med. 1956; 255: 57-65.

2. Mori M, Kuwabara S, Fukutake T, Hattori T. Plasmapheresis and Millar Fisher Syndrome: analysis of 50 Consecutive Cases. Journal of Neurology, Neurosurgery, Psychiatry 2002; 72: 680.

3. Odaka M, Yuki N, Hirata K. Anti-GQIb IgG antibody Syndrome: clinical and immunological range. J Neurol Neurosurgery Psychiatry 2001; 70: 50-5.

4. Ropper AH, Sammuels MA. Adams and Victor's Principles of Neurology, 6th ed. New York, United States: McGraw-Hill; 1987.p.1493-8.

5. Bradley WG, Daroff RB, Fenical GM, Jankovic J. Neurology in clinical Practice. 5th ed. USA:Elsevier;2008.p.2290-91.

6. Yuki N, Sato S, Tsuji S. Frequent Presence of anti-GQIb antibody in Fisher's syndrome. Neurology 1993; 43: 414-7.

7. Chiba A, Knsonoki S, Obata H. Serum anti-GQIb IgG antibody is associated with ophthalmoplegia in Miller-Fisher Syndrome and Guillain-Barre Syndrome; clinical and immunohistochemical Studies. Neurology 1993; 43: 1911-7. 